\title{
Serum albumin and albuminuria predict the progression of chronic kidney disease in patients with newly diagnosed type 2 diabetes: a retrospective study
}

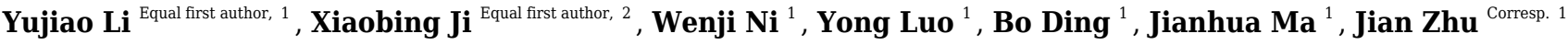 \\ 1 Department of Endocrinology, Nanjing First Hospital, Nanjing Medical University, Nanjing, Jiangsu, China \\ Department of Nephrology, Nanjing First Hospital, Nanjing Medical University, Nanjing, Jiangsu, China \\ Corresponding Author: Jian Zhu \\ Email address: drzhujian@hotmail.com
}

Background. Diabetes-related kidney disease is associated with end-stage renal disease and a high mortality rate. However, data on risk factors associated with kidney disease in patients with newly diagnosed type 2 diabetes mellitus (DM) remains insufficient. The aim of the present study was to identify the risk factors significantly associated with chronic kidney disease progression in patients with newly diagnosed type $2 \mathrm{DM}$.

Methods. We reviewed a total of 254 consecutive patients who were newly diagnosed with type 2 diabetes at Nanjing First Hospital from January to December 2014. They were observed for two years, and baseline and biochemical variables were used to identify significant predictors of kidney failure progression. Kidney failure progression was defined as $a \geq 30 \%$ increase in serum creatine level.

Results. The mean age of patients was 58.96 years, $37.4 \%$ were women, and $57.1 \%$ had hypertension. Kidney function progressed in 40 patients (15.75\%). Multivariable logistic regression analyses showed that serum albumin $(p=0.015)$ and microalbuminuria $(p<0.001)$ were associated with kidney failure progression in patients with newly diagnosed type $2 \mathrm{DM}$. Those with lower estimated glomerular filtration rate (eGFR; $30-60 \mathrm{ml} / \mathrm{min} / 1.73 \mathrm{~m}^{2}$ ) at baseline had lower serum albumin levels compared to those of patients with higher eGFR. The albuminuria levels were higher in patients with lower eGFR than in those with eGFR $\geq 90 \mathrm{ml} / \mathrm{min} / 1.73 \mathrm{~m}^{2}$. Receiver operating characteristic curve analysis showed that the area under the curve was $0.754(95 \% \mathrm{Cl} 0.670-0.0 .837)$.

Conclusions. The overall rate of chronic kidney disease progression is relatively high, and low serum albumin and high albuminuria levels are associated with kidney failure progression in newly diagnosed diabetic patients. 
1 Serum albumin and albuminuria predict the progression of chronic kidney disease in 2 patients with newly diagnosed type 2 diabetes: a retrospective study

3 Yu-jiao Li ${ }^{1}$, Xiao-bing Ji², Wen-ji Ni ${ }^{1}$, Yong Luo ${ }^{1}$, Bo Ding ${ }^{1}$, Jian-hua Ma ${ }^{1}$, Jian Zhu ${ }^{1}$

4 Yu-jiao Li and Xiao-bing Ji equally contributed as first authors.

$5{ }^{1}$ Department of Endocrinology, Nanjing First Hospital, Nanjing Medical University, Nanjing

6210029 China

$7 \quad{ }^{2}$ Department of Nephrology, Nanjing First Hospital, Nanjing Medical University, Nanjing

8210029 China

9 Corresponding Author:

10 Jian Zhu

11 Department of Endocrinology, Nanjing First Hospital, Nanjing Medical University, Nanjing

12210029 China

13 Email address: drzhujian@hotmail.com

E-mail Addresses:

16 Yu-jiao Li: drliyujiao@,outlook.com

17 Xiao-bing Ji: xbji@,njmu.edu.cn

18 Wen-ji Ni: drniwenji@hotmail.com

19 Yong Luo: luoyong132270@sina.com

20 BO Ding: dingbonanjing@sina.com

21 Jian-hua Ma: majianhua@china.com

\section{Abstract}

Background. Diabetes-related kidney disease is the leading cause of kidney failure worldwide. However, data on clinical markers predicting chronic kidney disease progression in patients with newly diagnosed type 2 diabetes mellitus (DM) remains insufficient. The purpose of this study was to identify the risk factors significantly associated with kidney function decline in newly diagnosed type 2 diabetic patients.

Methods. We reviewed a total of 254 consecutive patients who were newly diagnosed with type years, and baseline and biochemical variables were used to identify significant predictors of kidney function decline. Kidney function decline was defined as a $\geq 30 \%$ increase in serum creatinine level.

Results. The mean age of patients was 58.96 years, $37.4 \%$ were women, and $57.1 \%$ had hypertension. Kidney function decline occurred in 40 patients $(15.75 \%)$. Multivariable logistic regression analyses showed that serum albumin $(p=0.015)$ and albuminuria $(p<0.001)$ were associated with kidney function decline in patients with newly diagnosed type $2 \mathrm{DM}$. Those with lower estimated glomerular filtration rate (eGFR; 30-60 ml/min/1.73 $\mathrm{m}^{2}$ ) at baseline had lower 
serum albumin levels compared to those of patients with higher eGFR. The albuminuria levels were higher in patients with lower eGFR than in those with eGFR $\geq 90 \mathrm{ml} / \mathrm{min} / 1.73 \mathrm{~m}^{2}$. Receiver operating characteristic curve analysis showed that the area under the curve of albumin combined with albuminuria was 0.754 (95\% CI [0.670,0.837]).

Conclusions. The overall rate of chronic kidney disease progression is relatively high, and low serum albumin and high albuminuria levels are associated with kidney function decline in newly diagnosed type 2 diabetic patients.

Subject: Type 2 diabetes mellitus, Chronic kidney disease

Key words: Type 2 diabetes mellitus, Chronic kidney disease, Albuminuria, Albumin

\section{Introduction}

Diabetes-related chronic kidney disease (CKD) has become more common than CKD related to glomerulonephritis in both the general population and a hospitalized urban population in China(Zhang, Long et al. 2016). Diabetic patients with CKD are at risk for progression of kidney disease to end-stage renal disease (ESRD), cardiovascular events, and mortality (Retnakaran, Cull et al. 2006, Anders, Huber et al. 2018). To prevent CKD progression, intensive glycaemic control, lifestyle modifications, and administration of renin-angiotensin system inhibitors are used for diabetic patients. However, the rate of CKD progression to ESRD has remained unchanged over the past two decades (William, Hogan et al. 2005, Anders, Huber et al. 2018),with approximately 20 out of 10,000 adults with diabetes mellitus (DM) developing ESRD per year. Those numerous complications, comorbidities, and medications, as well as renal replacement therapy associated with advanced CKD and ESRD impose an enormous economic burden on the healthcare system (D'Onofrio, Simeoni et al. 2017, Gerber, Cai et al. 2018, Duan, Wang et al. 2019). Therefore, there is a need for epidemiologic studies to identify potentially modifiable risk factors to prevent the progression of kidney disease.

DM has approached epidemic proportions over the past decade worldwide. In China, the prevalence of diabetes has sharply increased. In a 2013 survey, 4\% of adults have been previously diagnosed with diabetes ( $\mathrm{Hu}$ and Jia 2018);6.9\% of patients have received a new diagnosis according to the American Diabetes Association 2010 criteria. Several studies had identified some clinical markers predicting CKD progression in previously diagnosed diabetic patients (Retnakaran, Cull et al. 2006, Dunkler, Gao et al. 2015, Go, Yang et al. 2018, Zhang, Ye et al. 2019). However, data on the risk factors related to kidney function decline in newly diagnosed type $2 \mathrm{DM}$ remain insufficient. Therefore, we undertook this retrospective observational study to examine the risk factors in this patient population.

\section{Materials and Methods}

\section{Study population}

We conducted a retrospective analysis on the clinical data of patients who were newly diagnosed with type 2 DM in Nanjing First Hospital from January 2014 to December 2014. Patients were 
excluded if they had the following: (1) acute complications of DM on admission, such as diabetic ketoacidosis; (2) severe infectious diseases on admission, fever, urinary tract infection, haematuria, glomerulonephritis; (3) severe cardiovascular diseases, such as stroke, myocardial infarction, coronary artery bypass grafting, percutaneous coronary intervention, and heart failure; (4) severely impaired liver function; (5) psychiatric disorders or were pregnant or planning to conceive; (6) cognitive disorders, alcoholism, or history of drug abuse; (7) acute kidney injury upon admission and/or at the end of follow-up. Patients lost to follow-up or with incomplete data were also excluded. The patients ranged in age from 18 to 86 years. All patients had the same medical team for treatment and care. The study protocol was approved by the Institutional Review Board at Nanjing First Hospital, Nanjing Medical University, which waived the requirement for written informed consent from patients.

\section{Data collection}

Data from all participants were collected anonymously. Type $2 \mathrm{DM}$ was diagnosed according to the American Diabetes Association 2010 criteria (Tankova 2007). Biochemical measurements were performed at the clinical laboratory at Nanjing First Hospital using a Beckman AU5800 clinical chemistry analyzer (Beckman Coulter, California, United States). Estimated glomerular filtration rate (eGFR) was calculated for each patient using the Modification of Diet in Renal Disease Study equation (Ma, Zuo et al. 2006): eGFR $\left(\mathrm{ml} / \mathrm{min} / 1.73 \mathrm{~m}^{2}\right)=175 \times \mathrm{SCr}$ $1.234 *$ age- $0.179 * 0.79$ (if female). Albuminuria levels were measured at the same laboratory by a chemiluminescent method using the IMMULITE 2000XPi immunoassay system (Shimadzu, Kyoto, Japan). The results were divided into quartiles ( $>300,100-300,30-100$, and $<30 \mathrm{mg} / 24 \mathrm{~h}$ ) and assigned a value $(4,3,2,1)$. The diagnosis of acute kidney injury was based on a serum creatine level increase of $\geq 0.3 \mathrm{mg} / \mathrm{dL}(26.52 \mu \mathrm{mol} / \mathrm{L})$ or $\geq 1.5$ - to twofold from baseline level and a urine output of $<0.5 \mathrm{~mL} / \mathrm{kg} / \mathrm{h}$ for more than six hours.

\section{Outcomes}

Kidney function decline was defined as a $\geq 30 \%$ increase in serum creatinine level. Patients whose serum creatine levels were increased because of acute kidney injury were excluded.

\section{Statistical analysis}

Data were analysed with PASW Statistics 18 software (SPSS (Hong Kong) Ltd., Hong Kong, China). Continuous variables were expressed as mean \pm standard error of the mean. Statistical significance of differences between groups were determined by t-test or one-way analysis of variance. Ordinal data were expressed as M (Q1, Q3), and a Wilcoxon rank sum test was used for comparisons between the two groups. Proportions were compared using the chi-squared test and Fisher's exact test when the numbers were small. All variables were first analysed using univariate analysis. Variables with a p-value $<0.1$ in the univariate analysis were then entered into a multivariable logistic regression analysis model to determine their net effects on kidney function decline. Odds ratios and their 95\% confidence intervals (CIs) were used to assess the independent contribution of prognostic factors. A p-value $<0.05$ was considered statistically significant. 


\section{Results}

\section{Demographic and clinical characteristics of patients}

116 The incidence of kidney function decline was $15.75 \%(n=40)$. The baseline demographics and 117 clinical characteristics of the patients are listed in Table 1. The mean age of the progression and 118 non-progression group were $62.90 \pm 16.21$ and $58.22 \pm 14.33$ years, respectively, and $62.6 \%$ of the

119 patients were male $(n=159)$. The mean body mass index of all enrolled was $25.21 \pm 3.53$, and

$12057.1 \%(n=145)$ of them had a history of hypertension. Kidney function decline was more likely

121 among patients with a history of hypertension and a larger hipline measurement.

\section{Biochemical variables of the patients}

123 The biochemical variables of the patients are listed in Table 2. The progression group had 124 significantly lower haemoglobin, total protein, albumin, and indirect bilirubin levels in blood; it 125 also had significantly higher glucose, triglyceride, low-density lipoprotein, and glycosylated 126 haemoglobin A1c levels.

\section{Potential risk factors for CKD progression in the multivariate logistic regression}

128 The multivariable logistic regression analysis was used to identify the risk factors for kidney function decline, and the results are listed in Table 3. After adjusting for confounders, both

130 serum albumin and albuminuria were associated with kidney function decline in patients with newly diagnosed type 2 DM.

\section{Associations between serum albumin, albuminuria and eGFR}

133 Those with lower eGFR level $\left(30-60 \mathrm{ml} / \mathrm{min} / 1.73 \mathrm{~m}^{2}\right)$ at baseline had lower serum albumin 134 compared to those of patients with an eGFR $60-90 \mathrm{ml} / \mathrm{min} / 1.73 \mathrm{~m}^{2}$ and eGFR $\geq 90 \mathrm{ml} / \mathrm{min} / 1.73$

$135 \mathrm{~m}^{2}$ (Figure 1). The albuminuria levels were higher in patients with lower eGFR level than in 136 those with eGFR $\geq 90 \mathrm{ml} / \mathrm{min} / 1.73 \mathrm{~m}^{2}$ (Figure 2).

\section{Receiver operating characteristic (ROC) curve estimation}

138 An ROC curve was created by plotting sensitivity against specificity at various threshold 139 settings. The $\mathrm{x}$-axis represents 1 -specifity, and the $\mathrm{y}$-axis represents sensitivity, the model was 140 well calibrated, and the area under the ROC curve is shown in Figure 3 and Table 4. The area 141 under the ROC curve of albumin was 0.702 (95\% CI [0.662, 0.782]), that of albuminuria was $1420.708(95 \%$ CI $[0.608,0.808])$, and that of albumin combined with albuminuria was $0.754(95 \%$ 143 CI $[0.670,0.837])$.

\section{Discussion}

145 The present study was undertaken to investigate the risk factors associated with kidney function 146 decline in newly diagnosed type 2 DM patients. A few studies had examined the risk factors for 147 CKD progression in diabetic patients (Retnakaran, Cull et al. 2006, Dunkler, Gao et al. 2015, 
148 Shih, Chuang et al. 2020). However, all patients were diagnosed with DM before enrolment in 149 these studies; therefore, the results could interfere with the use of medicine and lifestyle 150 modifications, which makes those studies prone to bias. Early predictions and delay of disease progression are of great concern in the clinical management of patients with type 2 DM. Our study demonstrated the potential risk factors that need to be managed in newly diagnosed type 2 DM patients.

In the present study, we observed an association between albuminuria and kidney function decline in type $2 \mathrm{DM}$ patients, and the predictive power of albuminuria for progressive kidney disease has previously been shown in diabetic patients (Spoelstra-de Man, Brouwer et al. 2001, Araki, Haneda et al. 2008). Several studies have found that many patients already have structural changes in kidney biopsy after the development of albuminuria(Looker, Mauer et al. 2018, Looker, Mauer et al. 2019), suggesting that albuminuria is a marker of established nephropathy rather than being a predictor of diabetic nephropathy. In our study, the albuminuria level is higher in the eGFR 30-60 group than in the eGFR $\geq 90$ group at baseline; the small sample size may have contributed to the lack of significant difference between patients in the eGFR 60-90 and eGFR $\geq 90$ groups. A recent study also showed that, in American Indians with type 2 DM, urine albumin to creatinine ratio reflects the progression of early structural glomerular lesions rather than early GFR decline (Looker, Mauer et al. 2019). It is generally acknowledged that progression of albuminuria may well represent a progression of early vascular disease, eventually leading to a cardiovascular end point. Thus, it cannot sufficiently emphasize the importance of albuminuria in diabetic patients.

We found that serum albumin was associated with kidney function decline in newly diagnosed diabetic patients independent of albuminuria. Previous studies have shown that low albumin levels are strongly associated with cardiovascular disease, heart failure, and mortality in vulnerable populations (Akirov, Masri-Iraqi et al. 2017, Gotsman, Shauer et al. 2019). Limited research has linked low serum albumin to kidney function decline (Lang, Scherzer et al. 2014, Jiang, Wang et al. 2020). Few studies have demonstrated that serum albumin is an important component of a multi-marker predictive model for progression to ESRD (Keane, Zhang et al. 2006, Lang, Katz et al. 2018). In our study, the serum albumin level was lower in the eGFR 3060 group than in the eGFR $\geq 90$ and eGFR 60-90 groups at baseline. A recent study showed that lower serum albumin levels are strongly and independently associated with the decline in kidney function in elderly individuals (Lang, Katz et al. 2018). Low albumin level could reflect an impairment in nutritional status or, in a blood dilution, be a marker that shows kidney damage or the progression of kidney failure. The potential mechanisms underlying these associations are unclear.

183 Only a few studies have so far reported that other risk factors independently predict CKD 184 progression in diabetic patients. De Cosmo et al (De Cosmo, Viazzi et al. 2015) found that mild hyperuricemia is strongly associated with the risk of CKD in patients with type 2 diabetes. Other risk factors include but are not limited to eGFR and hypertension (Retnakaran, Cull et al. 2006, Dunkler, Gao et al. 2015, Novak, Mucsi et al. 2016). We did not confirm this association, which may be explained by the difference in the race of patients enrolled.

189 Our study has several limitations. First, the study was a retrospective, single-centre study, thus 
191 accordingly, the observed significant differences may have been due to the small number of

192 patients in the progression group.

\section{Conclusion}

194 Low serum albumin and high albuminuria levels are strongly associated with CKD progression

195 in patients with newly diagnosed type 2 DM. Additional prospective studies in humans are

196 required to confirm this relationship and to understand the underlying mechanisms.

\section{Acknowledgments}

198 The authors would like to thank the patients enrolled in this study.

199

200

201

202

203

204

205

206

207

208

209

210

211

212

213

214

215

216

217

218

219

220

221

222

223

224

225

226

227

228

229

230

231

232

233

234

235

236

237

\section{References}

Akirov, A., H. Masri-Iraqi, A. Atamna and I. Shimon (2017). "Low Albumin Levels Are Associated with Mortality Risk in Hospitalized Patients." American Journal of Medicine 130(12): 1465.e1411-1465.e1419.DOI 10.1016/j.amjmed.2017.07.020

Anders, H. J., T. B. Huber, B. Isermann and M. Schiffer (2018). "CKD in diabetes: diabetic kidney disease versus nondiabetic kidney disease." Nat Rev Nephrol 14(6): 361-377.DOI 10.1038/s41581-018-0001-y

Araki, S., M. Haneda, D. Koya, A. Kashiwagi, T. Uzu and R. Kikkawa (2008). "Clinical impact of reducing microalbuminuria in patients with type 2 diabetes mellitus." Diabetes Research and Clinical Practice $\mathbf{8 2}$ Suppl 1: S54-58.DOI 10.1016/j.diabres.2008.09.031

D'Onofrio, G, M Simeoni, P Rizza, M Caroleo, M Capria, G Mazzitello, T Sacco, E Mazzuca, MT Panzino, A Cerantonio, C Segura-Garcia, M Andreucci, P De Fazio and G Fuiano (2017). "Quality of life, clinical outcome, personality and coping in chronic hemodialysis patients." Renal Failure 39(1): 45-53.DOI 10.1080/0886022x.2016.1244077

De Cosmo, S., F. Viazzi, A. Pacilli, C. Giorda, A. Ceriello, S. Gentile, G. Russo, M. C. Rossi, A. Nicolucci, P. Guida, D. Feig, R. J. Johnson and R. Pontremoli (2015). "Serum Uric Acid and Risk of CKD in Type 2 Diabetes." Clinical Journal of the American Society of Nephrology 10(11): 1921-1929.DOI 10.2215/cjn.03140315

Duan, J., C. Wang, D. Liu, Y. Qiao, S. Pan, D. Jiang, Z. Zhao, L. Liang, F. Tian, P. Yu, Y. Zhang, H. Zhao and Z. Liu (2019). "Prevalence and risk factors of chronic kidney disease and diabetic kidney disease in Chinese rural residents: a cross-sectional survey." Scientific Reports 9(1): 10408.DOI 10.1038/s41598-019-46857-7

Dunkler, D., P. Gao, S. F. Lee, G. Heinze, C. M. Clase, S. Tobe, K. K. Teo, H. Gerstein, J. F. Mann, R. Oberbauer, Ontarget and Origin Investigators (2015). "Risk Prediction for Early CKD in Type 2 Diabetes." Clinical Journal of the American Society of Nephrology 10(8): 1371-1379.DOI 10.2215/CJN.10321014

Gerber, C., X. Cai, J. Lee, T. Craven, J. Scialla, N. Souma, A. Srivastava, R. Mehta, A. Paluch, A. Hodakowski, R. Frazier, M. R. Carnethon, M. S. Wolf and T. Isakova (2018). "Incidence and Progression of Chronic Kidney Disease in Black and White Individuals with Type 2 Diabetes." Clinical Journal of the American Society of Nephrology 13(6): 884-892.DOI 10.2215/CJN.11871017

Go, A. S., J. Yang, T. C. Tan, C. S. Cabrera, B. V. Stefansson, P. J. Greasley, J. D. Ordonez and C. K. D. Outcomes Study Kaiser Permanente Northern California (2018). "Contemporary rates and predictors of fast progression of chronic kidney disease in adults with and without diabetes mellitus." BMC Nephrology 19(1): 146.DOI 10.1186/s12882-018-0942-1

Gotsman, I., A. Shauer, D. R. Zwas, I. Tahiroglu, C. Lotan and A. Keren (2019). "Low serum albumin: A significant predictor of reduced survival in patients with chronic heart failure." Clinical Cardiology 42(3): 365-372.DOI 10.1002/clc.23153

Hu, C. and W. Jia (2018). "Diabetes in China: Epidemiology and Genetic Risk Factors and Their Clinical Utility in Personalized Medication." Diabetes 67(1): 3-11.DOI 10.2337/dbi17-0013

Jiang, C., B. Wang, Y. Li, L. Xie, X. Zhang, J. Wang, Y. Yu, Y. Song, M. Liang, G. Wang, J. Li, Y. Zhang, L. Liu, C. Liu, G. Tang, Y. Huo, X. Xu and X. Qin (2020). "U-shaped association between serum albumin 
238

239

240

241

242

243

244

245

246

247

248

249

250

251

252

253

254

255

256

257

258

259

260

261

262

263

264

265

266

267

268

269

270

271

272

273

274

275

276

277

278

279

280

281

282

283

284

and development of chronic kidney disease in general hypertensive patients." Clinical Nutrition 39(1): 258264.DOI 10.1016/j.clnu.2019.02.002

Keane, W. F., Z. Zhang, P. A. Lyle, M. E. Cooper, D. de Zeeuw, J. P. Grunfeld, J. P. Lash, J. B. McGill, W. E. Mitch, G. Remuzzi, S. Shahinfar, S. M. Snapinn, R. Toto and B. M. Brenner (2006). "Risk scores for predicting outcomes in patients with type 2 diabetes and nephropathy: the RENAAL study." Clinical Journal of the American Society of Nephrology 1(4): 761-767.DOI 10.2215/cjn.01381005

Lang, J., R. Katz, J. H. Ix, O. M. Gutierrez, C. A. Peralta, C. R. Parikh, S. Satterfield, S. Petrovic, P. Devarajan, M. Bennett, L. F. Fried, S. R. Cummings, M. J. Sarnak and M. G. Shlipak (2018). "Association of serum albumin levels with kidney function decline and incident chronic kidney disease in elders." Nephrol Dial Transplant 33(6): 986-992.DOI 10.1093/ndt/gfx229

Lang, J., R. Scherzer, P. C. Tien, C. R. Parikh, K. Anastos, M. M. Estrella, A. G. Abraham, A. Sharma, M. H. Cohen, A. W. Butch, M. Nowicki, C. Grunfeld and M. G. Shlipak (2014). "Serum albumin and kidney function decline in HIV-infected women." American Journal of Kidney Diseases 64(4): 584-591.DOI 10.1053/j.ajkd.2014.05.015

Looker, H. C., M. Mauer and R. G. Nelson (2018). "Role of Kidney Biopsies for Biomarker Discovery in Diabetic Kidney Disease." Advances in Chronic Kidney Disease 25(2): 192-201.DOI 10.1053/j.ackd.2017.11.004

Looker, H. C., M. Mauer, P. J. Saulnier, J. L. Harder, V. Nair, C. M. Boustany-Kari, P. Guarnieri, J. Hill, C. A. Esplin, M. Kretzler, R. G. Nelson and B. Najafian (2019). "Changes in Albuminuria But Not GFR are Associated with Early Changes in Kidney Structure in Type 2 Diabetes." Journal of the American Society of Nephrology 30(6): 1049-1059.DOI 10.1681/asn.2018111166

Ma, Y. C., L. Zuo, J. H. Chen, Q. Luo, X. Q. Yu, Y. Li, J. S. Xu, S. M. Huang, L. N. Wang, W. Huang, M. Wang, G. B. Xu and H. Y. Wang (2006). "Modified glomerular filtration rate estimating equation for Chinese patients with chronic kidney disease." Journal of the American Society of Nephrology 17(10): 2937-2944.DOI 10.1681/asn.2006040368

Novak, M., I. Mucsi, C. M. Rhee, E. Streja, J. L. Lu, K. Kalantar-Zadeh, M. Z. Molnar and C. P. Kovesdy (2016). "Increased Risk of Incident Chronic Kidney Disease, Cardiovascular Disease, and Mortality in Patients With Diabetes With Comorbid Depression." Diabetes Care 39(11): 1940-1947.DOI 10.2337/dc160048

Retnakaran, R., C. A. Cull, K. I. Thorne, A. I. Adler, R. R. Holman and Ukpds Study Group (2006). "Risk factors for renal dysfunction in type 2 diabetes: U.K. Prospective Diabetes Study 74." Diabetes 55(6): 18321839.DOI 10.2337/db05-1620

Shih, H. M., S. M. Chuang, C. C. Lee, S. C. Liu and M. C. Tsai (2020). "Addition of Metabolic Syndrome to Albuminuria Provides a New Risk Stratification Model for Diabetic Kidney Disease Progression in Elderly Patients." Scientific Reports 10(1): 6788.DOI 10.1038/s41598-020-63967-9

Spoelstra-de Man, A. M., C. B. Brouwer, C. D. Stehouwer and Y. M. Smulders (2001). "Rapid progression of albumin excretion is an independent predictor of cardiovascular mortality in patients with type 2 diabetes and microalbuminuria." Diabetes Care 24(12): 2097-2101.DOI 10.2337/diacare.24.12.2097

Tankova, T. (2007). "Current criteria for the diagnosis of diabetes mellitus: 2006 WHO recommendations." Endokrinologya 12: 171-179.DOI

William, J., D. Hogan and D. Batlle (2005). "Predicting the development of diabetic nephropathy and its progression." Advances in Chronic Kidney Disease 12(2): 202-211.DOI 10.1053/j.ackd.2005.02.001

Zhang, D., S. Ye and T. Pan (2019). "The role of serum and urinary biomarkers in the diagnosis of early diabetic nephropathy in patients with type 2 diabetes." PeerJ 7: e7079.DOI 10.7717/peerj.7079

Zhang, L., J. Long, W. Jiang, Y. Shi, X. He, Z. Zhou, Y. Li, R. O. Yeung, J. Wang, K. Matsushita, J. Coresh, M. H. Zhao and H. Wang (2016). "Trends in Chronic Kidney Disease in China." New England Journal of Medicine 375(9): 905-906.DOI 10.1056/NEJMc1602469

Peer) reviewing PDF | (2020:11:55478:2:0:NEW 6 May 2021) 
Table $\mathbf{1}$ (on next page)

Baseline characteristics of individuals

RAAS: Renin-Angiotensin-Aldosterone system 


\begin{tabular}{lcccc}
\hline & $\begin{array}{c}\text { Non-Progression } \\
(\mathrm{n}=214)\end{array}$ & $\begin{array}{c}\text { Progression } \\
(\mathrm{n}=40)\end{array}$ & $\begin{array}{c}\text { Total } \\
(\mathrm{n}=254)\end{array}$ & p-value \\
\hline Sex(male) & 139 & 20 & 159 & 0.07 \\
Age $($ years $)$ & $58.22 \pm 14.33$ & $62.90 \pm 16.21$ & $58.96 \pm 14.70$ & 0.06 \\
BMI $\left(\mathrm{Kg} / \mathrm{m}^{2}\right)$ & $25.12 \pm 3.34$ & $25.68 \pm 4.46$ & $25.21 \pm 3.53$ & 0.35 \\
Waistline $(\mathrm{cm})$ & $88.42 \pm 8.70$ & $90.03 \pm 9.85$ & $88.67 \pm 8.89$ & 0.30 \\
Hipline(cm) & $94.01 \pm 7.52$ & $96.48 \pm 9.14$ & $94.40 \pm 7.83$ & 0.06 \\
Systolic pressure & $130.20 \pm 14.91$ & $138.50 \pm 17.50$ & $131.50 \pm 15.60$ & $<0.01$ \\
(mmHg) & & & & \\
Diastolic pressure & $78.73 \pm 9.74$ & $82.28 \pm 10.86$ & $79.29 \pm 9.98$ & 0.04 \\
(mmHg) & & $75.65 \pm 8.62$ & $77.44 \pm 7.87$ & 0.12 \\
Heart rate(beat/min) & $77.77 \pm 7.71$ & & & \\
Comorbidities & & 31 & 145 & $<0.01$ \\
Hypertension & 114 & 9 & 62 & 0.80 \\
Smoking & 53 & 6 & 31 & 0.56 \\
Drinking & 25 & 7 & 50 & 0.71 \\
Coronary heart disease & 43 & 14 & 69 & 0.23 \\
Diabetic retinopathy & 55 & 25 & 127 & 0.09 \\
Use of RAAS inhibitor & 102 & & &
\end{tabular}




\section{Table 2 (on next page)}

Biochemical variables of the individuals

$\mathrm{Hb}$ : haemoglobin, TSH: thyroid stimulating hormone, FT3:free triiodothyronine, FT4: free thyroxine, FBG $\square$ fasting blood glucose, HDL: high-density lipoprotein, LDL: low-density lipoprotein, HbAlc: glycosylated haemoglobin Alc 


\begin{tabular}{|c|c|c|c|c|}
\hline & $\begin{array}{l}\text { Non-Progression } \\
(\mathrm{n}=214)\end{array}$ & $\begin{array}{l}\text { Progression } \\
(n=40)\end{array}$ & $\begin{array}{l}\text { Total } \\
(\mathrm{n}=254)\end{array}$ & p-value \\
\hline $\mathrm{Hb}(\mathrm{g} / \mathrm{L})$ & $134.98 \pm 16.67$ & $127.95 \pm 13.99$ & $133.87 \pm 16.46$ & 0.01 \\
\hline TSH(mIU/L) & $2.11 \pm 1.88$ & $1.88 \pm 2.13$ & $2.08 \pm 1.92$ & 0.48 \\
\hline FT3(pmol/L) & $4.37 \pm 1.14$ & $4.11 \pm 0.49$ & $4.33 \pm 1.07$ & 0.15 \\
\hline FT4(pmol/L) & $15.95 \pm 5.84$ & $15.56 \pm 1.95$ & $15.89 \pm 5.42$ & 0.68 \\
\hline Total protein(g/L) & $68.83 \pm 6.56$ & $66.39 \pm 4.73$ & $68.44 \pm 6.36$ & 0.03 \\
\hline $\operatorname{Albumin}(\mathrm{g} / \mathrm{L})$ & $41.13 \pm 3.61$ & $38.92 \pm 2.88$ & $40.77 \pm 3.59$ & $<0.01$ \\
\hline Direct bilirubin $(\mu \mathrm{mol} / \mathrm{L})$ & $4.05 \pm 2.11$ & $3.96 \pm 1.99$ & $4.03 \pm 2.09$ & 0.80 \\
\hline Indirect bilirubin $(\mu \mathrm{mol} / \mathrm{L})$ & $8.67 \pm 4.60$ & $7.26 \pm 3.85$ & $8.45 \pm 4.51$ & 0.07 \\
\hline Creatine(mol/L) & $82.66 \pm 29.78$ & $80.75 \pm 38.38$ & $82.20 \pm 31.26$ & 0.72 \\
\hline Uric $\operatorname{acid}(\mu \mathrm{mol} / \mathrm{L})$ & $340.83 \pm 108.58$ & $343.98 \pm 102.87$ & $342.16 \pm 107.51$ & 0.90 \\
\hline $\mathrm{FBG}(\mathrm{mmol} / \mathrm{L})$ & $11.58 \pm 7.53$ & $13.93 \pm 7.67$ & $11.94 \pm 7.60$ & 0.07 \\
\hline HbA1c(\%) & $8.74 \pm 2.12$ & $9.77 \pm 2.21$ & $8.90 \pm 2.16$ & $<0.01$ \\
\hline $\begin{array}{l}\text { Total cholesterol } \\
(\mathrm{mmol} / \mathrm{L})\end{array}$ & $4.84 \pm 1.03$ & $5.06 \pm 1.28$ & $4.88 \pm 1.07$ & 0.23 \\
\hline Triglyceride(mmol/L) & $2.19 \pm 2.18$ & $3.13 \pm 4.12$ & $2.34 \pm 2.59$ & 0.03 \\
\hline HDL(mmol/L) & $1.31 \pm 0.35$ & $1.33 \pm 0.31$ & $1.31 \pm 0.34$ & 0.66 \\
\hline LDL(mmol/L) & $2.65 \pm 0.63$ & $2.91 \pm 1.02$ & $2.69 \pm 0.71$ & 0.03 \\
\hline $\begin{array}{l}\text { Albuminuria } \\
(\mathrm{mg} / 24 \mathrm{~h})\end{array}$ & $56.73 \pm 141.89$ & $282.10 \pm 394.84$ & $92.22 \pm 218.52$ & $<0.01$ \\
\hline
\end{tabular}

2 


\section{Table 3(on next page)}

Results of the multivariable analysis to determine the factors for kidney function decline 
1

\begin{tabular}{lccccc}
\hline Variable & \multirow{2}{*}{ Coefficient } & Standard & Statistic & p-value & OR $(95 \%$ CI $)$ \\
\hline Albumin & -0.123 & 0.051 & 5.872 & 0.015 & $0.884(0.801,0.977)$ \\
Albuminuria & 0.003 & 0.001 & 15.053 & $<0.001$ & $2.18(1.558,3.050)$ \\
\hline
\end{tabular}

2 
Table 4 (on next page)

ROC curve estimation 
1

\begin{tabular}{lcccc}
\hline Variable & AUC & Standard error & OR $(95 \%$ CI $)$ & P value \\
\hline Albumin & 0.702 & 0.041 & $0.702(0.662,0.782)$ & $<0.001$ \\
Albuminuria & 0.708 & 0.051 & $0.708(0.608,0.808)$ & $<0.001$ \\
Combined & 0.754 & 0.051 & $0.754(0.670,0.837)$ & $<0.001$ \\
\hline
\end{tabular}

2 
Figure 1

Relationship between serum albumin and eGFR category in 254 patients at baseline

*: $p<0.05$ eGFR 30-59 VS eGFR 60-89.9, \#: $p<0.05$ eGFR 30-59 VS eGFR $\geq 90$

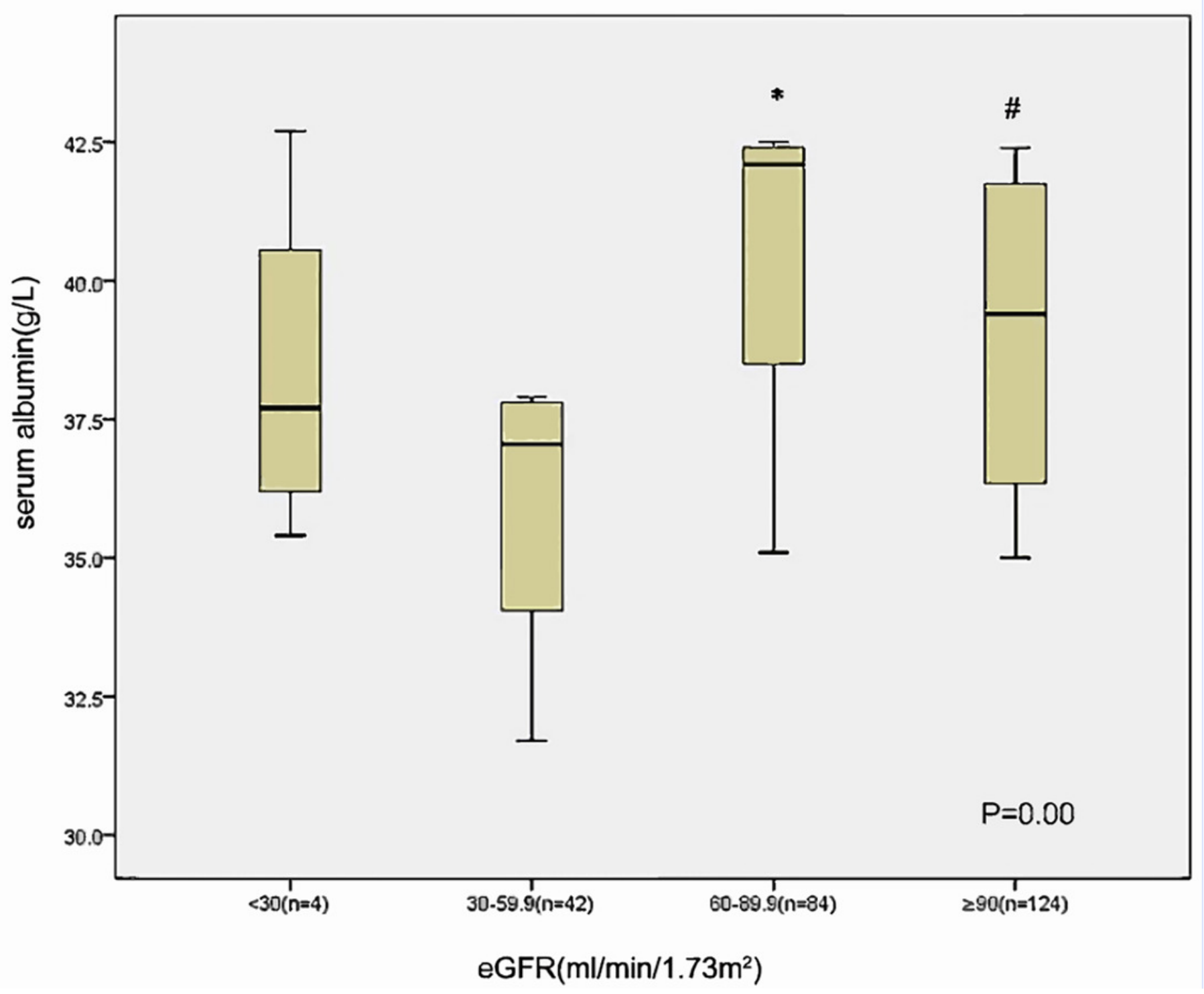


Figure 2

Relationship between albuminuria and eGFR category in 254 patients at baseline

*: $p<0.05$ eGFR $30-59$ VS eGFR $\geq 90$

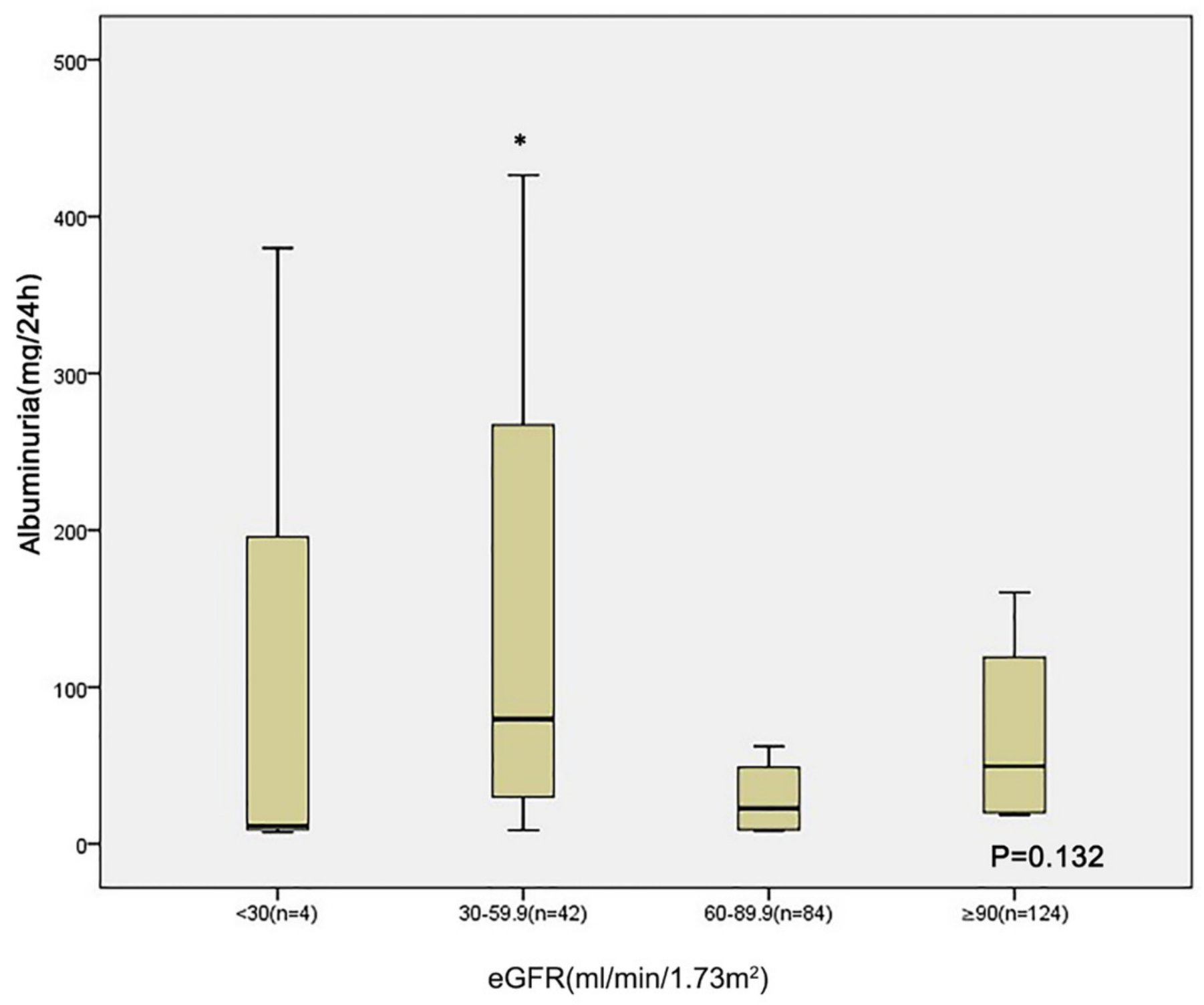


Figure 3

ROC curve estimation

(A) The AUC of albumin was $0.702(-0.662,0.782)$, (B) the AUC of albuminuria was 0.708 $(0.608,0.808)$, and $(C)$ the AUC of albumin combined with albuminuria was $0.754(95 \% \mathrm{Cl}$ 0.670-0. 0.837).

A

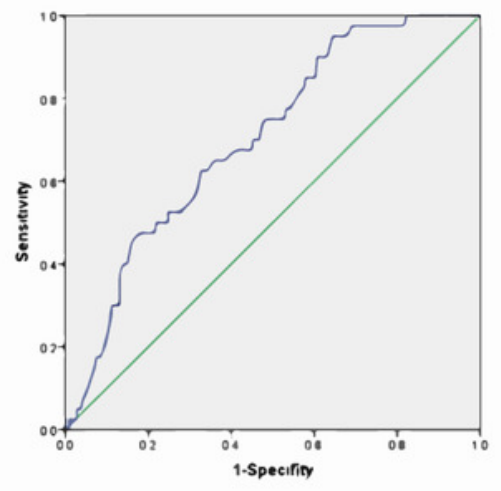

B

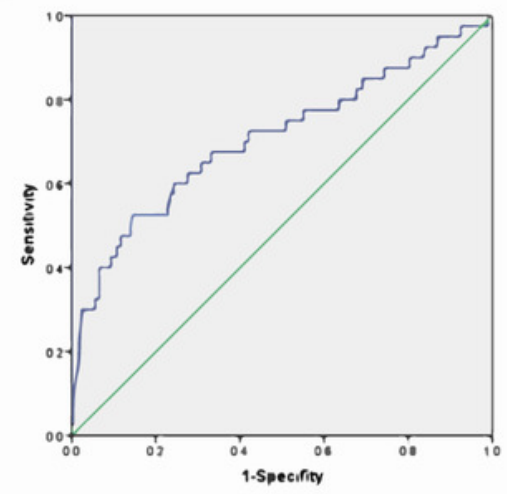

C

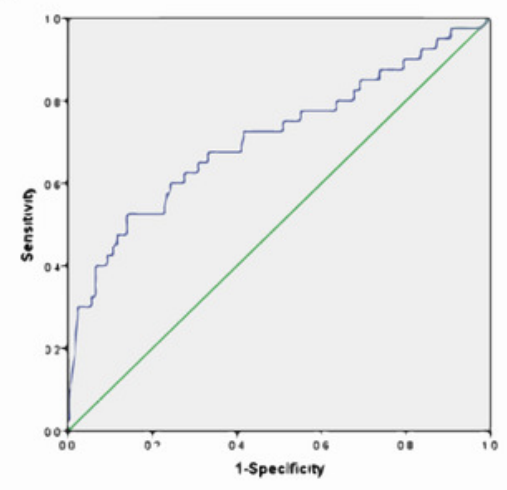

